Lívia de Almeida Barros Bertuluccl ${ }^{1}$ Flavia Maria Barros Guimarães Pereira ${ }^{2}$ Ana Emília Figueiredo de Oliveira ${ }^{1,2}$ Luciane Maria Oliveira Brito ${ }^{1}$ FERNANDA FERREIRA LOPES ${ }^{1,2}$

Artigo Original

Palavras-chave
Osteoporose
Pós-menopausa
Doenças periodontais
Keywords
Osteoporosis
Postmenopause
Periodontal diseases

Correspondência

Fernanda Ferreira Lopes Universidade Federal do Maranhão Programa de Pós-Graduação em Saúde Materno Infant Praça Goncạlves Dias, $n 21$ - Prédio de Medicina (IIA) - $2^{\circ}$ Andar - Centro (EP: $65020-240$

São Lứs (MA), Brasil

Recebido

04/05/2012

Aceito com modificacōes $06 / 10 / 2012$

\section{Doença periodontal em mulheres na pós- menopausa e sua relação com a osteoporose}

\author{
Periodontal disease in women in post-menopause and its \\ relationship with osteoporosis
}

\section{Resumo}

OBJETIVO: Verificar a relação entre periodontite e osteoporose em um estudo caso-controle sobre a condição periodontal das mulheres na pós-menopausa. MÉTODOS: A amostra foi composta por 99 mulheres na pós-menopausa, divididas em três grupos: osso normal (Gn), osteopenia (Gpenia) e osteoporose (Gporose), com 45, 31 e 23 casos, respectivamente. A categorização da massa óssea foi aferida pela absorciometria de dupla emissão com raios X na área lombar (L2-L4), e pela avaliação da densidade mineral óssea. Os índices de nível de inserção clínica (NIC), sangramento gengival (IG), de placa (IP) e profundidade de sondagem (PS) foram obtidos de todas as participantes, por apenas um examinador. Foi utilizado o programa BioEstat 2.0 para análise dos dados com os testes paramétricos análise de variância (ANOVA) e teste de Bonferroni, empregando-se o nível de significância de 5\%. RESULTADOS: O grupo de mulheres com osteoporose apresentou o maior percentual de presença da doença periodontal, com maior média do NIC (2,6 $\pm 0,4 \mathrm{~mm})$, assim como PS $(2,8 \pm 0,6 \mathrm{~mm}), \operatorname{IG}(72,8 \pm 25,9 \mathrm{~mm})$ e $\mathbb{P}(72,9 \pm 24,2 \mathrm{~mm})$. Após a realização do tratamento estatístico, observou-se que houve diferença significativa para a situação periodontal, principalmente entre os grupos $G$ n e Gporose $(p=0,01)$ e entre os grupos Gpenia e Gporose (p=0,03). CONCLUSÃO: A osteoporose pode ter uma influência na condição periodontal, por haver relação entre periodontite e osteoporose em mulheres na pós-menopausa.

\section{Abstract}

PURPOSE: To investigate the relationship between periodontitis and osteoporosis, using a case-control study about periodontal status of postmenopausal women. METHODS: A total of 99 postmenopausal women were divided into three groups: normal bone (Gn, n=45), osteopenia (Gpenia, n=31) and osteoporosis (Gporosis, $n=23$ ). The categorization of bone mass was measured by dual energy absorptiometry with X-rays in the lumbar spine (L2-L4), by assessing bone mineral density. Clinical attachment level (CAL), gingival bleeding index (GI), plaque index (PI), and probing depth (PD) were determined in all participants by a single examiner. The data were submitted to BioEstat 2.0 software through parametric analysis of variance (ANOVA) and the Bonferroni test, with the level of significance set at 5\%. RESULTS: Women with osteoporosis presented the highest percentage of periodontal disease, with higher average CAL $(2.6 \pm 0.4 \mathrm{~mm})$ and PD $(2.8 \pm 0.6 \mathrm{~mm}), \mathrm{Gl}(72.8 \pm 25.9 \mathrm{~mm})$ and PI $(72.9 \pm 24.2 \mathrm{~mm})$. Statistical analysis revealed a significant difference in periodontal situation between $\mathrm{G}$ and Gporosis $(\mathrm{p}=0,01)$ and between Gpenia and Gporosis $(p=0,03)$. CONCLUSION: Osteoporosis may have an influence on periodontal condition, based on the relation between periodontitis and osteoporosis in postmenopausal women.
Trabalho realizado no Programa de Pós-Graduação em Saúde Materno Infantil da Universidade Federal do Maranhão - UFMA São Luís (MA), Brasil.

'Programa de Pós-Graduação em Saúde Materno Infantil, Universidade Federal do Maranhão - UFMA - São Luís (MA), Brasil. 2Programa de Pós-Graduação em Odontologia, Universidade Federal do Maranhão - UFMA - São Luís (MA), Brasil.

Conflito de interesses: não há 


\section{Introdução}

Na pós-menopausa, a osteoporose juntamente com a fase senil do indivíduo tem sido amplamente reconhecida, nas duas últimas décadas, como um importante problema de saúde pública, sendo a mais comum doença ósseo-metabólica que atinge mulheres nessa fase. No entanto, não se pode deixar de lado as causas secundárias, como as afecções de origem endócrino-metabólicas (hereditárias ou não), reumatológicas, digestivas, renais, neoplásicas e uso de medicações que interferem com o equilíbrio do cálcio, tais como anticonvulsivantes, corticosteroides, antiácidos entre outras. Portanto, por ser uma doença sistêmica, a osteoporose pode afetar também ossos maxilares ${ }^{1-3}$.

A doença periodontal é caracterizada pela presença de lipopolissacarídeos e endotoxinas derivadas dos microrganismos (Gram-negativos) que, por sua vez, ativam várias células do hospedeiro a liberar citocinas inflamatórias como a interleucina 1 (IL-1). A IL-1ß é um dos mais potentes mediadores de reabsorção óssea, estimulando a diferenciação e ativação dos osteoclastos e inibindo também a formação óssea ${ }^{4}$.

Vários estudos vêm sendo desenvolvidos para determinar a associação existente entre osteoporose e a doença periodontal ${ }^{5,6}$; apesar disso, os resultados ainda são controversos ${ }^{7}$.

Embora a etiologia da osteoporose pós-menopausal e a periodontite sejam diferentes, a perda óssea ocorre nas duas doenças, as quais compartilham várias características $^{8}$. Como os receptores de estrógenos são expressos em células ósseas e imunes, foi levantada a hipótese de que a deficiência de estrogênio pode influenciar na remodelação óssea em sítios com processos inflamatórios ${ }^{9}$, uma vez que as células do ligamento periodontal expressam receptores específicos para estrogênios ${ }^{10}$.

Sugere-se que fatores sistêmicos responsáveis pela osteoporose podem interagir com fatores locais (doença periodontal), aumentando o padrão de perda óssea alveolar ${ }^{1}$. Por ser uma doença multifatorial, a osteoporose é apontada como um dos fatores de riscos para doença periodontal, mas os estudos existentes na literatura com população de mulheres na pós-menopausa são controversos ${ }^{7}$, o que justifica a realização desta pesquisa, cujo objetivo foi analisar quantitativamente, através de estudo caso-controle, a condição periodontal das mulheres na pós-menopausa e sua relação com a osteoporose.

\section{Métodos}

O presente trabalho consiste de uma pesquisa do tipo transversal analítica, que teve como grupo de estudo mulheres na pós-menopausa atendidas no Hospital Universitário da Universidade Federal do Maranhão através da Unidade Materno Infantil, totalizando 99 mulheres na pós-menopausa. A amostra foi de conveniência e o tamanho amostral foi calculado com base nos resultados de Gomes-Filho et al. ${ }^{4}$, empregando o poder de teste de $85 \%$ e nível alfa de $5 \%$, quando se obteve o número ideal de 98 indivíduos para serem incluídos na amostra. Como critério de inclusão foi adotada a confirmação do ciclo menstrual cessado por mais de um ano, com no mínino 40 anos de idade e sem uso de Terapia de Reposição Hormonal (TRH), e como critérios de exclusão: história de diabetes melittus e tabagismo.

Todas as pacientes assinaram termo de consentimento livre e esclarecido para a participação no estudo, que foi aprovado pelo Comitê de Ética em Pesquisa do Hospital Universitário da Universidade Federal do Maranhão, sob o parecer consubstanciado de número 172/08. A presente pesquisa foi conduzida de acordo com a Declaração de Helsinque revisada em 2008 e não possui conflitos de interesse.

O diagnóstico da osteoporose foi baseado no escore $\mathrm{T}$ da densitometria óssea, com dados obtidos de uma população normal de referência, pela avaliação da coluna lombar, segmento L2-L4, e/ou colo do fêmur, sendo considerado o valor mais baixo identificado em uma das duas zonas do esqueleto ${ }^{11}$. Nas mulheres pós-menopausadas, considera-se normal o valor da densidade mineral óssea (DMO) de 0 a -1 desvio padrão (DP) do encontrado na população de adultos jovens ou do pico de massa óssea (escore T); entre -1,0 e -2,5 DP, aplica-se o termo osteopenia. O diagnóstico da osteoporose é aplicado quando o valor da DMO for menor do que -2,5 DP, de acordo com os critérios definidos pela Organização Mundial da Saúde ${ }^{12}$.

O exame da condição periodontal consistiu da aplicação dos seguintes índices: índice de sangramento gengival (IG), idealizado por Ainamo e Bay, e índice de placa (IP), de O'Leary et al. ${ }^{13,14}$. O IG e o IP foram realizados com o auxílio de uma sonda periodontal (sonda tipo Willians), que circundou delicadamente a margem gengival por vestibular, palatino ou lingual, mesial e distal de todos os dentes presentes para obtenção do valor por pessoa e a média de cada grupo de estudo.

A medida da extensão da perda das estruturas de inserção foi feita pelas medições da profundidade de bolsa (distância entre a margem gengival e o fundo de sulco/ bolsa periodontal) e de perda de inserção clínica (distância entre o limite amelocementário e o fundo de sulco/bolsa periodontal $)^{15}$. Cada medida clínica foi obtida em seis sítios periodontais (mesiovestibular, mediovestibular, distovestibular, mesiolingual, mediolingual e distolingual) de todos os dentes em cada paciente, exceto os terceiros molares, para obtenção da média dos dentes, observando-se as medidas de profundidade à sondagem e nível clínico de inserção ${ }^{16}$. Para a obtenção dessas medidas clínicas foi utilizada a sonda milimetrada tipo Willians, paralela ao 
longo eixo do dente. O exame clínico foi realizado a cego por um único examinador, ou seja, o avaliador não teve conhecimento da situação óssea sistêmica das pacientes.

O banco de dados foi construído em planilha Excel e utilizado o software BioEstat 2.0 para a análise estatística dos dados. Os procedimentos estatísticos clássicos no estudo de associação entre variáveis foram empregados, através de testes paramétricos análise de variância (ANOVA) e teste de Bonferroni, empregando-se o nível de significância de 5\%. Para verificar a distribuição da doença periodontal entre os grupos de estudo (osso normal $=\mathrm{Gn}$; osteopenia $=\mathrm{Gpenia}$; osteoporose $=$ Gporose $)$ foi aplicado o teste do $X^{2}(a=0,05)$. A progressão da doença periodontal foi medida em termos da perda de inserção periodontal, por melhor mensurar o dano periodontal no indivíduo, sendo classificada como presença de periodontite quando havia perda de inserção clínica maior ou igual a $4 \mathrm{~mm}$ em pelo menos dois sítios ou um sítio com perda de inserção maior ou igual a $5 \mathrm{~mm}^{16,17}$.

\section{Resultados}

Como resultado desta pesquisa, tem-se que a média de idade das participantes foi de 55,8 anos. Todas as mulheres eram parcialmente dentadas, com média de 12,1 dentes, sendo a média de 56,7\% das superfícies dentais com placa bacteriana e $53,1 \%$ com sangramento gengival à sondagem.

Após o exame clínico, todas as participantes foram alocadas em grupos de estudo, conforme a situação sistêmica, tendo sido a amostra composta por 45 mulheres com osso normal (Gn), 31 com osteopenia (Gpenia) e 23 com osteoporose (Gporose). A média de idade das participantes dos grupos Gn, Gpenia e Gporose foi, respectivamente, de 53,3; 56,3 e 59,5 anos, sem diferença significativa $(\mathrm{p}>0,05)$ por meio da análise de variância (teste de Kruskal-Wallis; $\boldsymbol{\alpha}=0,05$ ).
A Tabela 1 mostra comparações dos parâmetros periodontais entre os grupos de estudo, assim como as médias e as significâncias estatísticas. O exame periodontal revelou que as mulheres na pós-menopausa do grupo Gpenia apresentaram menor média do NIC $(2,00 \pm 0,49 \mathrm{~mm})$, assim como PS (2,06 $\pm 0,49 \mathrm{~mm}), \mathrm{IG}(45,5 \pm 25,6 \mathrm{~mm}) \mathrm{e}$ IP $(48,0 \pm 26,3 \mathrm{~mm})$. Os valores das variáveis NIC, PS, IG e IP foram submetidos ao tratamento estatístico (teste de Bonferroni), por meio do qual se detectou haver diferença significante, principalmente entre os grupos Gn e Gporose e entre os grupos Gpenia e Gporose.

A Tabela 2 expressa a distribuição das mulheres na pós-menopausa, de acordo com a situação óssea sistêmica e a presença ou não de periodontite, em que $34,78 \%$ das mulheres com osteoporose apresentavam periodontite versus $11,11 \%$ das mulheres com osso normal, evidenciando que a periodontite é significativamente mais comum em mulheres com osteoporose.

Tabela 2. Distribuição das mulheres na pós-menopausa, de acordo com os grupos de estudo e a presença ou não de periodontite

\begin{tabular}{|c|c|c|c|c|c|c|}
\hline \multirow{3}{*}{$\begin{array}{l}\text { Grupos de } \\
\text { estudo }\end{array}$} & \multirow{2}{*}{\multicolumn{2}{|c|}{ Total }} & \multicolumn{4}{|c|}{ Periodontite } \\
\hline & & & \multicolumn{2}{|c|}{ Presente } & \multicolumn{2}{|c|}{ Ausente } \\
\hline & n & $\%$ & $n$ & $\%$ & n & $\%$ \\
\hline $\mathrm{Gn}^{\mathrm{a}}$ & 45 & 100 & 5 & 11,1 & 40 & 88,8 \\
\hline Gpenia ab & 31 & 100 & 4 & 12,9 & 27 & 87,1 \\
\hline Gporose c & 23 & 100 & 8 & 34,7 & 15 & 65,2 \\
\hline Total & 99 & 100 & 17 & 17,1 & 82 & 82,8 \\
\hline \multicolumn{7}{|c|}{$\begin{array}{l}\text { Letras minúsculas iguais indicam ausência de diferença estatística, segundo teste } \\
\text { do } x^{2} \text { : partição }(a=0,05) \text {. }\end{array}$} \\
\hline Partição & \multicolumn{2}{|c|}{ Grupos em comparação } & $x^{2}$ & \multicolumn{2}{|c|}{ GL } & Valor $p$ \\
\hline 1 & \multicolumn{2}{|c|}{ Gn $x$ Gpenia } & 2,1 & \multicolumn{2}{|c|}{1} & 0,1 \\
\hline 2 & \multicolumn{2}{|c|}{ Gpenia x Gporose } & 4,4 & \multicolumn{2}{|c|}{1} & 0,03 \\
\hline 3 & \multicolumn{2}{|c|}{ Gn x Gporose } & 5,5 & \multicolumn{2}{|c|}{1} & 0,01 \\
\hline Total & \multicolumn{2}{|c|}{ Gn x Gpenia x Gporose } & 6,5 & \multicolumn{2}{|c|}{2} & $<0,05$ \\
\hline
\end{tabular}

Tabela 1. Comparações dos parâmetros periodontais entre os grupos de estudo pelo teste de Bonferroni, após a ANOVA com um fator, expressando as diferenças das médias e as significâncias estatísticas

\begin{tabular}{|c|c|c|c|c|c|c|c|c|}
\hline Parâmetro & Grupo & Média & DP & $\mathbf{F}$ & Valor $p$ & \multicolumn{2}{|c|}{ Bonferroni B } & Valor $p$ \\
\hline \multirow{4}{*}{ NIC } & Gn & 2,1 & 0,4 & \multirow{3}{*}{11,8} & \multirow{3}{*}{$<0,01$} & Gn x Gpenia & 0,2 & $>0,05$ \\
\hline & Gpenia & 2,0 & 0,4 & & & Gn $\times$ Gporose & 0,2 & $<0,05$ \\
\hline & Gporose & 2,6 & 0,4 & & & Gpenia x Gporose & 0,3 & $<0,05$ \\
\hline & Gn & 2,3 & 0,6 & \multirow{3}{*}{10,5} & \multirow{3}{*}{$<0,01$} & Gn x Gpenia & 0,2 & $<0,05$ \\
\hline \multirow[t]{2}{*}{ OS } & Gpenia & 2,0 & 0,4 & & & Gn $x$ Gporose & 0,2 & $<0,05$ \\
\hline & Gporose & 2,8 & 0,6 & & & Gpenia x Gporose & 0,2 & $<0,05$ \\
\hline \multirow[t]{3}{*}{ IG } & Gpenia & 45,5 & 25,6 & \multirow[t]{3}{*}{7,9} & \multirow[t]{3}{*}{$<0,01$} & Gn x Gporose & 11,6 & $<0,05$ \\
\hline & Gporose & 72,8 & 25,9 & & & Gpenia x Gporose & 12,4 & $<0,05$ \\
\hline & Gn & 54,5 & 26,8 & & & Gn x Gpenia & 10,1 & $>0,05$ \\
\hline \multirow[t]{2}{*}{ IP } & Gpenia & 48,0 & 26,3 & \multirow[t]{2}{*}{6,2} & \multirow[t]{2}{*}{$<0,01$} & Gn x Gporose & 11,1 & $<0,05$ \\
\hline & Gporose & 72,9 & 24,2 & & & Gpenia x Gporose & 11,9 & $<0,05$ \\
\hline
\end{tabular}




\section{Discussão}

O presente estudo demonstrou que as mulheres na pós-menopausa do grupo Gporose apresentaram maior média do NIC, assim como de PS, IG e IP. Após tratamento estatístico encontrou-se relação significativa entre a condição periodontal e a situação óssea sistêmica do osso normal (Gn) e osteoporose (Gporose), assim como entre os grupos Gpenia e Gporose, ratificando evidências de associação entre osteopenia/osteoporose sistêmica e risco aumentado de doença periodontal, como citado em outros estudos ${ }^{18-21}$.

Estudos recentes ${ }^{22,23}$ indicam que a osteoporose pode influenciar a condição periodontal, pois as mulheres com osteoporose frequentemente apresentam quadro clínico com inflamação gengival mais intensa, bem como valores de perda de inserção clínica e recessão gengival mais elevados que as mulheres com densidade mineral óssea normal $^{22}$, indicando que a osteoporose pode ter uma influência na condição periodontal ${ }^{23}$, de modo semelhante ao registrado em nosso estudo.

Em contrapartida, a relação entre a densidade mineral óssea esquelética e a perda óssea alveolar interproximal e perda de inserção clínica também já foi registrada, embora de forma não significante ${ }^{24}$. Porém, ressalta-se que o diagnóstico precoce de densidade mineral óssea reduzida pode ser importante, pois pode prever um impacto negativo sobre os tecidos periodontais, sem deixar de lado a importância do controle de variáveis como terapia hormonal, tabagismo e status menopausal ${ }^{22}$. Esse fato fundamenta-se na hipótese de que a deficiência de estrogênio influencia na remodelação óssea em sítios com processos inflamatórios ${ }^{8}$, como observados na doença periodontal ${ }^{25}$. Na presente pesquisa, não havia mulheres em terapia de reposição hormonal, não sendo, portanto, avaliada a influência dessa variável sobre os resultados.

No presente estudo, observou-se que a diminuição da DMO em mulheres estava associada a valores mais elevados de nível de inserção clínica Tal evidência é amparada em dados sobre a suscetibilidade à doença periodontal em mulheres com redução na $\mathrm{DMO}^{19}$.

O baixo nível educacional foi apontado como um fator de risco à periodontite em mulheres osteoporóticas ${ }^{5}$; no entanto, a metodologia aplicada na presente pesquisa não investigou esse fator, apesar de, em ambos os estudos, terem sido incluídas mulheres na pós-menopausa, com idade acima de 50 anos, que estavam sendo atendidas em unidade de serviço de saúde pública.

Divergindo dos resultados de nossa pesquisa, não foi encontrada diferença significativa em relação ao sangramento gengival, profundidade de sondagem, recessão gengival e nível do osso alveolar entre as mulheres com densidade óssea mineral normal e as osteoporóticas ${ }^{26}$. Uma possível explicação para essa divergência pode estar alicerçada nas variáveis idade e tamanho da amostra, pois, em nosso estudo, as mulheres osteoporóticas apresentaram média de idade mais elevada, porém sem diferença significativa quando comparada aos grupos com osteopenia e osso normal. No entanto, é estabelecido que tanto a periodontite quanto a osteoporose estão associadas com a idade ${ }^{26}$.

Ausência de correlação entre nível de inserção clínica e densidade mineral óssea no primeiro exame periodontal já foi registrada em estudo coorte ${ }^{27}$, divergindo dos nossos resultados que evidenciaram significativa perda de inserção no grupo de mulheres com osteoporose. Vale ressaltar que, ao longo dos três anos, foram identificadas correlações fracas entre nível de inserção e densidade mineral óssea ${ }^{27}$, concordando-se que a osteopenia pode ser um fraco fator de risco para doença periodontal ${ }^{28}$.

Outros estudos ${ }^{7,17}$ também não evidenciaram a possível influência de mudanças na densidade mineral óssea sobre os tecidos periodontais. Provavelmente, essas diferenças na literatura se devem a diferentes metodologias empregadas. Um aspecto importante a ser considerado é o método de avaliação da densidade óssea mineral ${ }^{29}$.

Apesar de a presente pesquisa e de o estudo longitudinal sobre a associação entre a osteoporose e a doença periodontal em mulheres na pós-menopausa ${ }^{24}$ apresentarem delineamentos diferentes, desde o tamanho amostral até a análise dos resultados, em ambos os estudos foi ratificada a associação entre condição óssea sistêmica e doença periodontal. Após três anos, Lopes et $\mathrm{al}^{24}$ verificaram maior perda de inserção clínica periodontal em mulheres com osteopenia/ostoporose, enquanto na presente pesquisa transversal foi evidenciada média de nível de inserção clínica mais elevada e maior frequência de periodontite em mulheres osteoporóticas. Essa categorização dos diagnósticos de doença periodontal não havia sido realizada no estudo anterior ${ }^{24}$.

A perda no nível de inserção periodontal observada entre as mulheres com osteoporose, analisadas no presente estudo, ratifica a associação entre a osteoporose e um dos parâmetros de avaliação da doença periodontal, ou seja, o nível de inserção clínica ${ }^{30}$, confirmando assim a hipótese de que as mulheres na pós-menopausa com osteoporose apresentam maior probabilidade de ter doença periodontal do que as mulheres sem osteoporose 5 .

\section{Agradecimentos}

À Fundação de Amparo a Pesquisa e ao Desenvolvimento Científico e Tecnológico do Maranhão - FAPEMA pela concessão de bolsas de mestrado. 
1. López-Marcos JF, García-Valle S, García-lglesias AA. Periodontal aspects in menopausal women undergoing hormone replacement therapy. Med Oral Patol Oral Cir Bucal. 2005; 10(2): 132-41.

2. Taguchi A, Sanada M, Suei Y, Ohtsuka M, Nakamoto T, Lee $K$, et al. Effect of estrogen use on tooth retention, oral bone height, and oral bone porosity in Japanese postmenopausal women. Menopause. 2004;1 1(5):556-62.

3. Engebretson SP, Grbic JT, Singer R, Lamster IB. GCF IL-1 $\beta$ profiles in periodontal disease. J Clin Periodontol. 2002;29(1):48-53.

4. Gomes-Filho IS, Passos JS, Cruz SS, Vianna MI, Cerqueira EM, Oliveira DC, et al. The association between postmenopausal osteoporosis and periodontal disease. J Periodontol. 2007;78(9):1731-40.

5. Kulikowska-Bielaczyc E, Gołebiewska M, Preferansow E. The relationship between mineral status of the organism and the number of teeth present and periodontal condition in postmenopausal patients. Adv Med Sci. 2006;51 Suppl 1:130-3.

6. Pallos D, Ceschin A, Victor GA, Bulhões RC, Quirino MRS. Menopause: a risk factor for periodontal disease? Rev Bras Ginecol Obstet. 2006;28(5):292-7.

7. Lerner UH. Inflammation-induced bone remodeling in periodontal disease and the influence of post-menopausal osteoporosis. J Dent Res. 2006;85(7):596-607.

8. Lerner UH. Bone remodeling in post-menopausal osteoporosis. J Dent Res. 2006;85(7):584-95.

9. Cao M, Shu L, Li J, Su J, Zhang W, Wang Q, et al. The expression of estrogen receptors and the effects of estrogen on human periodontal ligament cells. Methods Find Exp Clin Pharmacol. 2007;29(5):329-35.

10. Cunha S. Fatores de risco de osteoporose feminina. Avaliação do risco segundo ORAl e realização de DEXA. Rev Port Clin Geral. $2005 ; 21(6): 537-43$.

11. Pinto Neto AM, Soares A, Urbanetz AA, Souza ACA, Ferrari AEM, Amaral B, et al. Consenso brasileiro de osteoporose 2002. Rev Bras Reumatol. 2002;42(6):343-54.

12. Ragghianti MS, Greghi SL, Lauris JR, Sant'Ana AC, Passanezi $E$. Influence of age, sex, plaque and smoking on periodontal conditions in a population from Bauru, Brazil. J Appl Oral Sci. 2004; 12(4):273-9.

13. Hofer D, Sahrmann P, Attin T, Schmidlin PR. Comparison of marginal bleeding using a periodontal probe or an interdental brush as indicators of gingivitis Int J Dent Hyg. 2011 ; 9(3): 211 1-5.

14. Nonnenmacher C. Parâmetros clínicos e radiográficos no diagnóstico periodontal. In: Oppermann RV, Rosing CK, coordenadores. Periodontia: ciência e clínica. São Paulo: Artes Médicas; 2001 . p. 23-29.

15. Borges-Yáñez SA, Maupomé G, Jimenéz-Garcia G. Validity and reliability of partial examination to assess severe periodontitis. J Clin Periodontol. 2004;31 (2):112-8.
16. Familli P, Cauley J, Suzuki JB, Weyant R. Longitudinal study of periodontal disease and edentulism with rates of bone loss in older women. J Periodontol. 2005;76(1):1 1-5.

17. Ronderos M, Jacobs DR, Himes JH, Pihlstrom BL. Associations of periodontal disease with femoral bone mineral density and estrogen replacement therapy: cross-sectional evaluation of US adults from NHANES III. J Clin Periodontol. 2000;27(10):778-86.

18. Mohammad AR, Hooper DA, Vermilyea SG, Mariotti A, Preshaw PM. An investigation of the relationship between systemic bone density and clinical periodontal status in post-menopausal AsianAmerican women. Int Dent J. 2003;53(3):121-5.

19. Tezal M, Wactawski-Wende J, Grossi SG, Ho AW, Dunford R, Genco $\mathrm{RJ}$. The relationship between bone mineral density and periodontitis in posmenopausal women. J Periodontol. 2000;71(9):1492-8.

20. Krejci $C B$, Bissada NF. Women's health issues and their relationship to periodontitis. J Am Dent Assoc. 2002;133(3):323-9.

21. Pepelassi E, Nicopoulou-Karavianni K, Archontopoulou AD, Mitsea A, Kavadella A, Tsiklakis K, et al. The relationship between osteoporosis and periodontitis in women aged 45-70 years. Oral Dis. 2012;18(4):353-9.

22. Gomes-Filho IS, Oliveira TJ, Passos JS, Cerqueira ED, da Cruz SS, Barreto $\mathrm{ML}$, et al. Effect of osteoporosis on periodontal therapy among post-menopausal women. Gerodontology. 2012 Mar 16. [Epub ahead of print].

23. Sultan N, Rao J. Association between periodontal disease and bone mineral density in postmenopausal women: A cross sectional study. Med Oral Patol Oral Cir Bucal. 2011 ; 16(3):e440-7.

24. Lopes FF, Loureiro FHF, Pereira AFV, Pereira ALA, Alves CMC. Association between osteoporosis and periodontal disease. Rev Bras Ginecol Obstet. 2008;30(8):379-83.

25. Lundström A, Jendle J, Stenström B, Toss G, Ravald N. Periodontal conditions in 70-year-old women with osteoporosis. Swed DentJ. 2001 ;25(3):89-96.

26. Pilgram TK, Hildebolt CF, Dotson M, Cohen SC, Hauser JF, Kardaris $\mathrm{E}$, et al. Relationships between clinical attachment level and spine and hip bone mineral density: data from healthy postmenopausal women. J Periodontol. 2002;73(1 1):298-301.

27. Weyant RJ, Pearlstein ME, Churak, AP, Forrest K, Famili P, Cauley JA. The association between osteopenia and periodontal attachment loss in older women. J Periodontol. 1999;70(9):982-91.

28. Rosetti EP, Sampaio LM, Zuza EP, Cioca SC, Toledo BEC. A influência da menopausa no desenvolvimento da doença periodontal: revisão de literatura. Periodontia. 2007;17(4):20-3.

29. Brennan RM, Genco RJ, Hovey KM, Trevisan M, Wactawski-Wende J. Clinical attachment loss, systemic bone density, and subgingival calculus in postmenopausal women. J Periodontol. 2007;78(1 1):2104-1 1.

30. Brennan RM, Genco RJ, Hovey KM, Trevisan M, Wactawski-Wende J. Clinical attachment loss, systemic bone density, and subgingival calculus in postmenopausal women. J Periodontol. 2007;78(1 1):2104-1 1. 